\title{
Comparative Evaluation Of Sphenopalatine Ganglion Block Versus Pregabalin For Treatment Of Postdural Puncture Headache Following Surgery Under Subarachnoid Block
}

\author{
Abhishek Verma ( $\nabla$ drabhishekverma5@gmail.com ) \\ Vardhman Mahavir Medical College \& Safdarjung Hospital \\ Rajkumar M P \\ Vardhman Mahavir Medical College \& Safdarjung Hospital \\ Anjali Kochhar \\ Vardhman Mahavir Medical College \& Safdarjung Hospital \\ Monika Gupta \\ Vardhman Mahavir Medical College \& Safdarjung Hospital \\ Rajpal Singh \\ Vardhman Mahavir Medical College \& Safdarjung Hospital \\ Krishan Kumar \\ Vardhman Mahavir Medical College \& Safdarjung Hospital
}

\section{Short Report}

Keywords: post dural puncture headache, sphenopalatine block, dural puncture, VAS score, spinal anaesthesia

Posted Date: February 28th, 2022

DOI: https://doi.org/10.21203/rs.3.rs-1374868/v1

License: (c) (i) This work is licensed under a Creative Commons Attribution 4.0 International License. Read Full License 


\section{Abstract}

Background: PDPH (postdural puncture headache) can be devastating for the patients and challenging for the clinicians at the same time. In many upcoming modalities of treatment of PDPH we compared sphenopalatine ganglion block and pregabalin among patients undergoing subarachnoid block.

Methods: ASA grade I and II patients who developed PDPH following surgery (both elective and emergency) under subarachnoid block were included. Patients with moderate to severe headache were randomized into two groups. Group SP-received SPGB and group P-received pregabalin 150mg. Pain relief was assessed by visual analogue score (VAS) at regular intervals. Inj. paracetamol $1 \mathrm{~g}$ IV was given as rescue analgesia. Follow up done for 72 hours or till pain relief.

Main Results: Mean VAS measured at 1 hour interval in group SP $(3.13 \pm 0.43)$ was significantly lower as compared to and group $P(6.03 \pm 0.99)$ with $p$ value $(p=0.001)$. Mean VAS at 12 hours in group $P$ was $2.70 \pm 0.75$ as compared to group SP $(3.40 \pm 1.03)$ and difference was statistically significant with $p$ value $(p=0.004)$. The requirement of rescue analgesia at 1 hour was found to be significantly lower in group SP $(p=0.0008)$ and at 12 -hour requirement was significantly lower in group P $(p=0.001)$. No difference was observed in VAS score or rescue analgesia at all other time intervals.

Conclusion: We conclude that any patient with moderate or severe PDPH, both the treatment modalities (sphenopalatine nerve block and pregabalin) are effective as SPG nerve block gives immediate relief and pregabalin provides sustained pain relief.

\section{Introduction:}

Post dural puncture headache (PDPH) is defined by the international classification of headache disorder as a headache occurring within 5 days after lumbar puncture, and being aggravated when standing or sitting and relieved when lying supine position ${ }^{1}$.

Development of PDPH may result in increased morbidity, prolonged hospital stays, increased cost, and patient dissatisfaction ${ }^{2}$. Autologous epidural blood patch (AEBP) is considered the gold standard treatment for $\mathrm{PDPH}$, with efficacy of up to $75 \%{ }^{3}$.

AEBP is an invasive procedure with risks of difficulty identifying the epidural space, inadvertent dural puncture, patient discomfort during the procedure, infection, and neurologic complications ${ }^{4}$. There is a constant search for non-invasive or minimally invasive alternatives. Sphenopalantine block is a recent such modality ${ }^{5}$. Reports on use of sphenopalatine ganglion block as treatment for PDPH has shown that SPG block is better than AEBP in terms of pain relief and complications associated with the procedure ${ }^{6}$.

Pregabalin is a gabapentanoid, which has been used in the treatment of neuropathic pain. The drug usually binds to alpha 2delta subunit of voltage gated calcium channels ${ }^{7}$. Recent studies Mahoori et al \& Bhattacharya et al show its effectiveness in $\mathrm{PDPH}^{8,9}$. 
To the best of our knowledge, there are no randomized studies comparing sphenopalatine ganglion block with pregabalin for the treatment of PDPH. Hence study planned with an aim to compare and evaluate sphenopalatine ganglion block versus pregabalin in relieving postdural puncture headache in patients following surgery under subarachnoid block.

\section{Patients And Methods:}

After getting clearance from ethical committee all patients ASA grade I and II who developed PDPH following surgery both elective and emergency under sub arachnoid block were included. Patients with previous history migraine, psychiatric illness, eclampsia, preeclampsia, accidental dural puncture were excluded.

A total of 1569 patients were screened in which about 158 patients experienced PDPH. After obtaining a written and informed consent all the patients were advised restricted activity along with intravenous hydration ringer lactate at $2 \mathrm{ml} / \mathrm{kg} / \mathrm{hr}$ and orally as per patient acceptance.

Patients with moderate to severe headache were randomized into two groups of 30 each, by computer generated random number enclosed in envelope as follows:

Group SP- received transnasal SPGB 4\%lignocaine

A long applicator with a cotton swab at the tip was soaked with $4 \%$ lignocaine solution. It was then inserted parallel to the floor of the nose until resistance is encountered. The swab was placed at the posterior pharyngeal wall superior to the middle turbinate. The applicator was retained in the nostril for 5-10 minutes and then removed. The procedure was similarly repeated in the other nostril.

Group P - received pregabalin $150 \mathrm{mg}$

Patients received $150 \mathrm{mg}$ pregabalin orally once, and followed for pain relief.

Headache was assessed by visual analogue score 0 -no pain $10=$ worst possible pain .

The time of intervention noted and VAS noted 1 hour after intervention and every six hours after intervention were noted. Injection paracetamol $1 \mathrm{~g} \mathrm{IV}$ used as rescue analgesic and requirement of rescue analgesia in every patient was noted. other symptoms such as neck stiffness and tinnitus were observed.

\section{STATISTICAL ANALYSIS:}

Sample size of 27.5 were calculated with $90 \%$ power of study and $5 \%$ level of significance based on results of a previous study Dr. Kochhar A, et al (2017) observed VAS of sphenopalatine block at 24 and 48 hours was 3.41 and 2.75 respectively ${ }^{10}$. In each study group to reduce the margin of error $10 \%$ loss of follow-up rounded to 30 in each group. Categorical variables were presented in number and percentage (\%) and continuous variables were presented as mean, SD and median. 
Normality of data was tested by Kolmogorov-Smirnov test. If the normality stood

rejected then non parametric test was used.

Quantitative variables were compared using ANOVA/Kruskal Wallis test between two groups and Unpaired t-test/Mann-Whitney Test (when the data sets were not normally distributed) between the two groups. Qualitative variables were compared using Chi-Square test /Fishers exact test, $p$ value of $<0.05$ was considered statistically significant.

The data was entered in MS EXCEL spreadsheet and analysis was done using the latest available Statistical Package for Social Sciences (SPSS) version.16.0(Chicago. Inc, USA)

\section{Results:}

In our study is among 1569 patients 158 patients had PDPH, out of which 98 were mild and 60 people were included in study 4 were severe and 56 were moderate.

The mean age of patients of SP and P group was $28.43 \pm 9.21$ and $26.90 \pm 5.31$ years respectively. There was no significant $(p>0.05)$ difference in age and were comparable between groups. Majority of patients of both SP $(86.7 \%)$ and P $(80 \%)$ groups were females. There was no significant $(p>0.05)$ difference in gender between the groups showing comparability of the groups in terms of gender. There was no significant difference in mean weight of group SP $(53.97 \pm 7.56 \mathrm{kgs})$ and group $\mathrm{P}(53.10 \pm 6.04 \mathrm{kgs})(\mathrm{p}>$ 0.05). We observed 109(68.3\%) patients were from obstetric population and 49 (31.7\%) were from other department. (Table 1).

Table 1

Demographic distribution between groups

\begin{tabular}{|llll|}
\hline Groups & $\begin{array}{l}\text { Age in years } \\
(\text { Mean } \pm \text { SD) }\end{array}$ & Gender & $\begin{array}{l}\text { Weight } \\
\text { (Mean } \pm \text { SD) }\end{array}$ \\
\hline $\mathrm{SP}(\mathrm{n}=30)$ & $28.43 \pm 9.21$ & $\begin{array}{l}\text { Male }-13.3 \% \\
\text { Female-86.7\% }\end{array}$ & $53.97 \pm 7.56$ \\
\hline $\mathrm{P}(\mathrm{n}=30)$ & $26.90 \pm 5.31$ & $\begin{array}{l}\text { Male }-20 \% \\
\text { Female }-80 \%\end{array}$ & $53.10 \pm 6.04$ \\
\hline $\mathrm{p}$-value & 0.43 & 0.48 & 0.62 \\
& (Unpaired t test) & (Chi square test) & (Unpaired t test) \\
\hline
\end{tabular}

There was no statistically significant difference in the baseline VAS among patients of group SP (5.63 \pm 0.80 ) versus group $P(6.03 \pm 0.99)$ with $p>0.05$ (Table 2$)$. 
Table 2

Comparison of baseline VAS between the groups

\begin{tabular}{|llll|}
\hline Time periods & $\begin{array}{l}\text { Group SP } \\
\text { VAS (mean } \pm \text { SD) }\end{array}$ & $\begin{array}{l}\text { Group P } \\
\text { VAS (mean } \pm \text { SD) }\end{array}$ & $\begin{array}{l}\text { p-value } \\
\text { (Unpaired t-test) }\end{array}$ \\
\hline Baseline & $5.63 \pm 0.80$ & $6.03 \pm 0.99$ & 0.09 \\
\hline 1 hour & $3.13 \pm 0.43$ & $6.03 \pm 0.99$ & 0.001 \\
\hline 6 hours & $3.03 \pm 0.76$ & $3.20 \pm 0.71$ & 0.38 \\
\hline 12 hours & $3.40 \pm 1.03$ & $2.70 \pm 0.75$ & 0.004 \\
\hline 18 hours & $2.73 \pm 0.86$ & $2.37 \pm 0.99$ & 0.13 \\
\hline 24 hours & $2.55 \pm 1.10$ & $1.95 \pm 0.95$ & 0.06 \\
\hline 30 hours & $2.33 \pm 0.86$ & $2.11 \pm 0.60$ & 0.53 \\
\hline
\end{tabular}

On comparison of VAS between two groups at 1 hour there was significant reduction in pain score in group SP $(3.13 \pm 0.43)$ as compared to group $P(6.03 \pm 0.99)$ with $p$ value $(0.001)$. There was no statistically difference observed in mean VAS at 6-hour time period. At 12-hour time period there was significant reduction in VAS in group P $(2.70 \pm 0.75)$ than group SP $(3.40 \pm 1.03)$ with $p$ value $(0.004)$ and then after reduced mean VAS in group P than group SP at every time interval (Table 2), but there was no statistically significant difference seen at 18, 24, 30 36-hour time interval. Figures (1)

The requirements of rescue analgesia at 1 hour interval were statistically significant in group $P$ with $p$ value (0.0008). There was no statistically difference observed in requirement of rescue analgesia at 6hour time period. At $12 \mathrm{hr}$ there was more requirement of rescue analgesia in group SP $(56.7 \%)$ than group P (16.7\%), with p value (0.001), after that no significant differences seen in time period $18,24,30$ 36 hours (Fig. 2)

There were no significant differences in both the group on comparing neck stiffness and tinnitus. In our observation, 8 patients in group P experienced dizziness (26.6\%). No side effects were observed in SP group.

\section{Discussion:}

Post dural puncture headache (PDPH) is defined by the international classification of headache disorder as a headache occurring within 5 days after lumbar puncture, and being aggravated when standing or sitting and relieved when lying supine position ${ }^{1}$.

In our study, PDPH was found to be more common in young age group, females and obstetric cases were more than non-obstetric cases. All these are the well-known risk factor for PDPH. PDPH is more common in young is due to the fact that with increasing age the dura may be less elastic and less likely to gape ${ }^{11}$. 
Women are at increased risk of PDPH (twice as men), due to physiological, anatomical, social, behavioral factors. They have increased perception of pain; hormonal factors may also contribute like high estrogen levels in serum and influence of tone in cerebral blood vessels ${ }^{12}$.

Obstetric patients are at higher risk because of the increased cerebrospinal fluid (CSF) pressure related to pregnancy, dehydration, blood loss, postpartum diuresis, hormona I imbalance, and increased peridural pressure $^{13,14}$.

We studied sphenopalantine block and compared it with pregabalin for treatment of PDPH. Results of our study show that patients who received SPG block got immediate pain relief with significant reduction in VAS score. The possible reason for this could be that SPGB uses $4 \%$ lignocaine, which is faster acting local anaesthetic and the pain relief was rapid.

Our findings are supported by various reports in the literature that demonstrate immediate relief of headache after SPG block. In a retrospective study by Patel et al ${ }^{6} 54.55 \%$ of the patients recovered from headache within half an hour of the SPG block and at one hour $63.64 \%$ of the patients were symptom free.

In a case report by Furtado et al, patient, a case of intra cranial hypertension, developed headache following excessive lumboperitoneal drain. Conservative management was started but failed to reduce pain. After SPG block patient got immediate and sustained relief for 24 hours $^{15}$.

We observed significant reduction in VAS in group P at 6- and 12-hour interval, findings of which are supported by Huseyinoglu $U$ et al in a study where 40 patients, who developed PDPH following spinal anaesthesia or after lumbar puncture were studied. They found that group which received pregabalin has significant reduction in VAS score, the requirement of NSAIDs and opioid analgesics were significantly reduced $^{16}$.

In a study by Bhattacharya et al, 150 patients were enrolled and randomized into three groups (pragabalin $150 \mathrm{mg}$, paracetamol $1000 \mathrm{mg}$ and both combined). All patients had significant pain relief in the symptoms. There was no significant difference in efficacy of pregabalin versus paracetemol. However, time taken for relief of head ache was significantly more in paracetamol group ${ }^{9}$.

Thus, Pregabalin is a promising pharmacological agent for the management of PDPH and its analgesic efficacy is secondary to presynaptic inhibition of the release of excitatory neurotransmitters in stimulated neurons, upregulation of alpha 2-delta subunit playing an important role in hypersensitization process $^{17,18}$.

We observed in our study initially requirement of rescue analgesia was significantly high in group P at 1 $\mathrm{hr}$ of observation. At $6 \mathrm{hrs}$, there was no difference seen in analgesic requirement between the groups. At 12 hours in group SP the patients required more rescue analgesia when compared to group P. 
This can be explained by pharmacokinetics of pregabalin which has an onset time of 1 hour. Pregabalin is well absorbed after oral administration with an average bio availability exceeding $90 \%$. It has a mean elimination half-life of approximately 6 hours in subjects with normal renal function? ${ }^{7}$.

The possible explanation to increased requirement of rescue analgesia at $12 \mathrm{hr}$ in SPG group lies in its mechanism of action. In transnasal SPGB block, the parasympathetic activity is blocked and thereby inhibiting the cerebral vasodilatation and patient gets symptomatic relief. As the effect of given local anaesthetic wanes away head ache reappears.

Pregabalin has its own side effects including drowsiness, dizziness, dry mouth lethargy and confusion. However, among those drowsiness and dizziness are most common when they are used as single dose. In our observation, 8 patients in group P experienced dizziness (26.6\%).

The observed incidence of dizziness and drowsiness by Paul F et al and Rahat Dahmardeh A et al after receiving single dose of pregabalin incidence was $(11-30 \%)^{19,20}$.

Adverse events of Transnasal SPGB are epistaxis and very rarely systemic local anaesthetic toxicity but in our observation, there was no such adverse events seen during study period.

Limitations of our study include inclusion of both obstetric and non-obstetric cases,exculsion of PDPH caused by accidental dural puncture, blinding was not possible due to nature of treatmentgroups.

Furthur studies are recommended combining pragabalin with SPG block to know the additive effects of these drugs in management of PDPH.

\section{Conclusion:}

We conclude that any patient with moderate or severe PDPH both the treatment modalities (sphenopalantine nerve block and pregabalin) are effective as SPG nerve block gives immediate relief and pregabalin provides sustained pain relief.

\section{Declarations:}

\section{DECLARATION OF PATIENT CONSENT}

The authors certify that they have obtained all appropriate patient consent forms. In the form the patient(s) has/have given his/her/their consent for his/her/their images and clinical information to be reported in the journal. The patients understand that their names and initials will not be published and due efforts will be made to conceal their identity but anonymity cannot be guaranteed. 


\section{References:}

1. Headache Classification Committee of the International Headache Society (IHS). The International Classification of Headache Disorders, 3rd edition. Cephalalgia2013;33:629-808.[1]

2. Syed S, Qayoom N, Naaz S, Mushtaq K, Mir SH, Bijli AH et al. Comparison of post-dural puncture headache- incidence and severity in obstetric patients after spinal anesthesia for cesarean section with 25G and 27G Quincke needle. Int J Res Med Sci. 2017;5:596-600.

3. Ylönen P, Kokki H. Epidural blood patch for management of postdural puncture headache in adolescents. Acta Anaesthesiol Scand 2002; 46: 794-8.

4. Paech M. Epidural blood patch-myths and legends. Can J Anaesth 2005;52:R1-5.

5. Kent S, Mehaffey G. Transnasal sphenopalatine ganglion block for the treatment of postdural puncture headache in obstetric patients. J Clin Anesth 2016; 34: 194-6.

6. Cohen S, Levin D, Mellender S, Zhao R, Patel P, Grubb W, Kiss G. Topical sphenopalatine ganglion block compared with epidural blood patch for post dural puncture headache Management in postpartum patients a retrospective review. Reg anesth Pain Med 2018;43:880-4.

7. Taylor C, Angelotti T, Fauman E. Pharmacology and mechanism of action of pregabalin: The calcium channel $a 2-\delta$ (alpha2-delta) subunit as a target for antiepileptic drug discovery. Epilepsy Research. 2007;73:137-150.

8. Mahoori A, Noroozinia H, Hasani E, Saghaleini H. Comparing the effect of pregabalin, gabapentin, and acetaminophen on post-dural puncture headache. Saudi J Anaesth 2014;8:374-7.

9. Bhattacharya D, Paul S, Naskar S, Mitra M, Mandal M. Comparison of the respective effects of paracetamol, pregabalin, and their combination in the treatment of postdural puncture headache following major gynecological surgery. Ain-Shams Journal of Anaesthesiology. 2016;9:387.

10. Kochhar A, Khanooja S, Yousuf I, Bhat M K, Bhat M. Evaluation of sphenopalatine block for treatment of postdural puncture headache following spinal anaesthesia: a randomized study. Ejpmr,2017;4(12):231-4.

11. Di Cianni S, Rossi M, Casati A, Cocco C, Fanelli G. Spinal anesthesia: an evergreen technique. Acta Biomed 2008; 79: 9-17.

12. Amorim J, Gomes de Barros M, Valença M. Post-dural (post-lumbar) puncture headache: Risk factors and clinical features. Cephalalgia. 2012;32(12):916-923.

13. Lybecker H, Møller JT, May O, Nielsen HK. Incidence and prediction of postduralpuncture headache. A prospective study of 1021 spinal anesthesia. Anesth Analg. 1990;70:389-94.

14. Vallejo MC, Mandell GL, Sabo DP, Ramanathan S. Postdural puncture headache: A randomized comparison of five spinal needles in obstetric patients. Anesth Analg. 2000;91:916-20.

15. Furtado I, Pinto M, Amorim P. Sphenopalatine Ganglion Block May Be an Efficient Treatment of Headache After Lumboperitoneal Shunt Placement. A \& A Practice. 2019;12(11):401-402.

16. Huseyinoglu U, Huseyinoglu N, Hamurtekin E, Aygun H, Sulu B. Effect of pregabalin on post-duralpuncture headache following spinal anesthesia and lumbar puncture. Journal of Clinical 
Neuroscience. 2011;18(10):1365-1368

17. Bansal A, Tewari A, Garg S, Gupta A. Pregabalin: Pharmacology and Use in Pain Management.J Anaesth Clin Pharmacol 2009; 25(3): 321-26

18. Luo ZD, Chaplan SR, Higuera ES, Sorkin LS, Stauderman KA, Williams ME, et al. Upregulation of Dorsal Root Ganglion a2 $\delta$ Calcium Channel Subunit and Its Correlation with Allodynia in Spinal Nerve-Injured Rats. J Neurosci. 2001;21(6):1868-75.

19. Paul F. White, Bureu Tufanogullari, Jimmie Taylor, Kevin Klein. The effect of pregabalin on preoperative anxiety and sedation levels: a dose ranging study Anesth Analg. 2009;108:1140-5.

20. Rahat Dahmardeh A, Moosavi A, Nasir-al-din Tabatabaei SM, Ordoni Avval J, Sistanizad M. The Effect of a Single Dose Oral Pregabalin on Hemodynamic Changes and Duration of Analgesia after Spinal Anesthesia in Orthopedic Surgeries of Tibial. Iran J Pharm Res. 2018;17:2-7

\section{Figures}

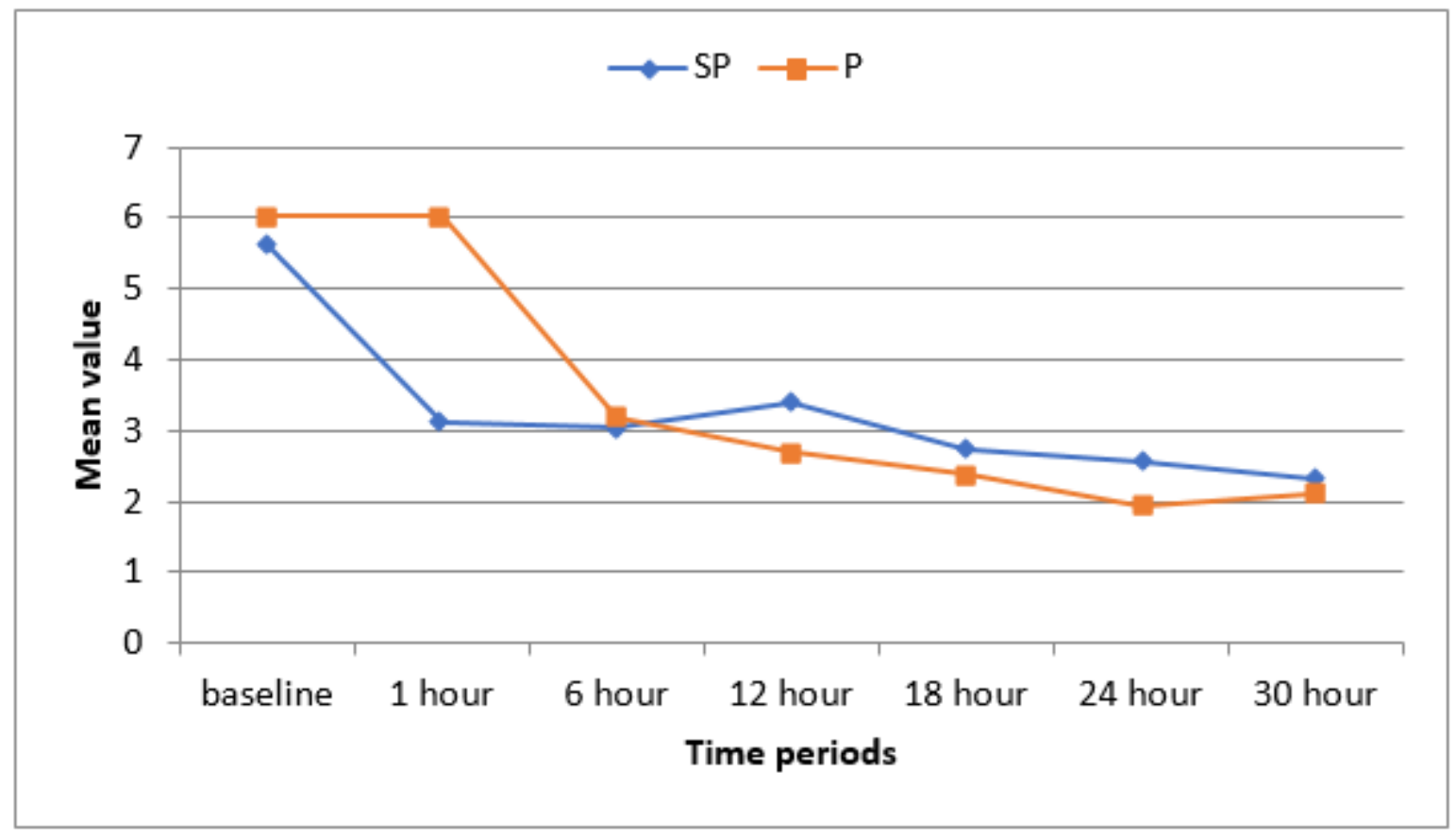

\section{Figure 1}

\section{Comparison of mean VAS between the groups}




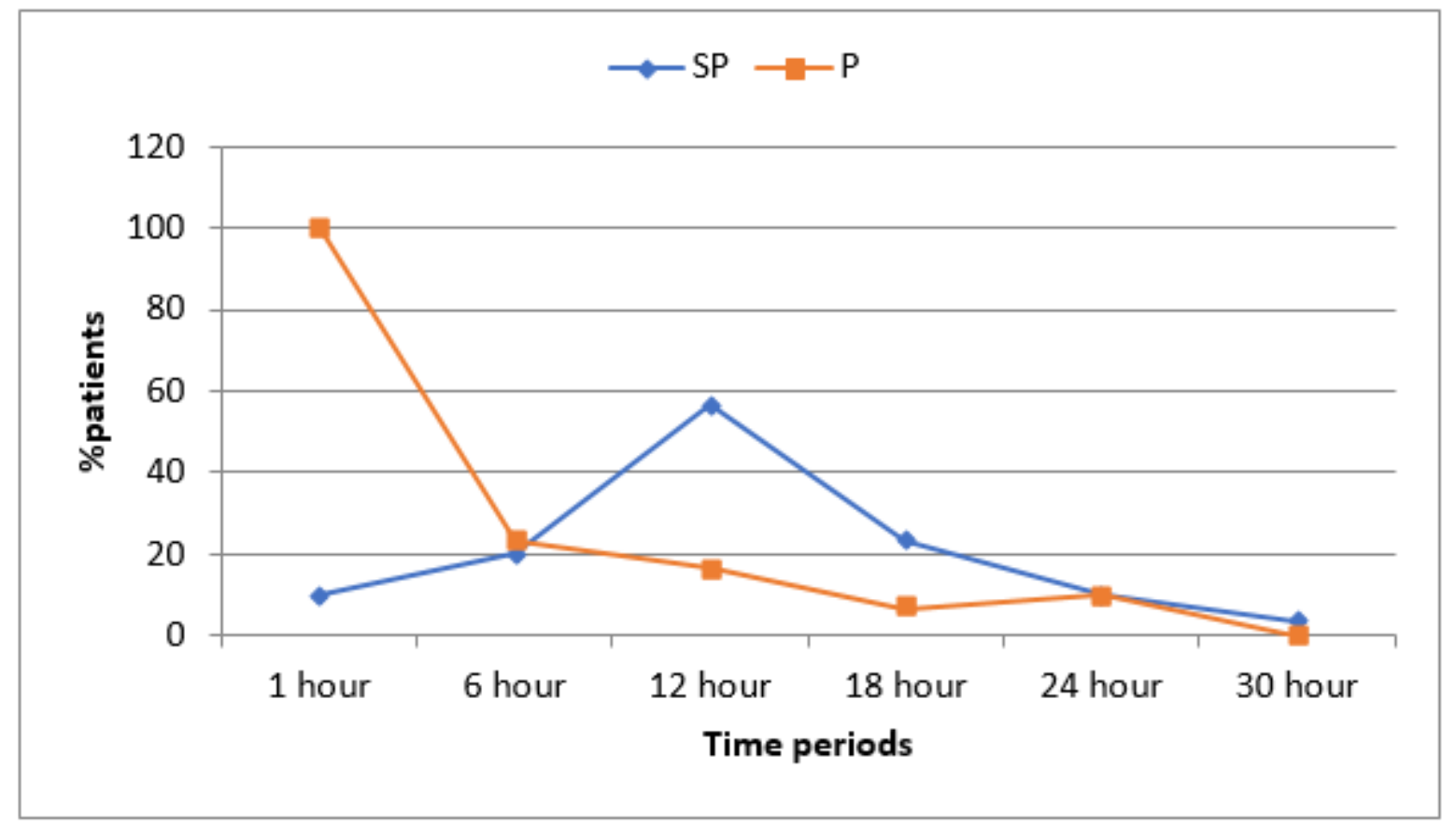

Figure 2

Comparison of rescue analgesia between the groups 